\title{
One-pot diastereoselective synthesis of new spiro indenoquinoxaline derivatives containing cyclopropane ring
}

\author{
Masoud Shaabanzadeh, ${ }^{a} *$ and Faranak Khabari ${ }^{\mathrm{b}}$ \\ ${ }^{a}$ Chemistry Department, Islamic Azad University, Damghan Branch, Zip Code 36716-39998, \\ Damghan, Iran \\ ${ }^{b}$ Chemistry Department, Islamic Azad University, Saveh Branch, P.O. Box 39187-366, \\ Saveh, Iran \\ E-mail: masoud.shaabanzadeh@gmail.com
}

\begin{abstract}
A one-pot procedure was introduced for the synthesis of some new spiro derivatives of indenoquinoxaline containing cyclopropane ring from chalcones. The chalcones 1a-e were reacted with hydrazine hydrate and then in situ with lead(IV) acetate (LTA) and two new diastereomers 2a-e and 3a-e were prepared. The compounds 2a-e diastereoselectively produced in higher yields than 3a-e.
\end{abstract}

Keywords: Spiro compounds, chalcones, LTA, indenoquioxalines, cyclopropane, diastereoselective reaction

\section{Introduction}

The cyclopropane ring is a main structural part in many synthetic and natural compounds that exhibits a wide range of biological activities from enzyme inhibition to antibiotic, herbicidal, antitumor, and antiviral properties. ${ }^{1,2}$ Some derivatives of cyclopropane have shown potent HIV antiviral activities as non-nucleoside reverse transcriptase inhibitors. ${ }^{3}$ Due to diversity of cyclopropane containing compounds with biological activity, chemists have tried to find novel and facile methods for synthesis of these compounds. ${ }^{4,5}$

Indenoquinoxaline derivatives are important classes of nitrogen containing heterocycles and they have applications in dyes and are useful intermediates in organic synthesis and have also been used as building blocks for the synthesis of organic semiconductors. ${ }^{6}$ More studies have discovered that these compounds exhibit diverse medical functions such as antimetabolism and antitubercular properties. $^{7}$

Many synthetic methods have been reported for the preparation of cyclopropanes such as intramolecular cyclization, addition of carbenes to olefins and Michael initiated ring closure 
(MIRC). ${ }^{1,8}$ In this study along our pervious works on the synthesis of spiro indenoquinoxalines and other biologically active compounds, ${ }^{9,}{ }^{10}$ we report a novel one-pot facile procedure for the synthesis of some spiro indenoquinoxaline-cyclopropane derivatives which directly prepared from chalcones of indenoquinoxaline.

\section{Results and Discussion}

Among the synthetic procedures for preparation of cyclopropane rings, the Michael initiated ring closure (MIRC) reaction of $\alpha, \beta$-unsaturated carbonyl compounds (like chalcones) with dimethylsulfoxonium methylides or Corey-Chaykovsky reaction is the well-known method. ${ }^{11,12}$ In this reaction, the cyclopropane ring forms between $\mathrm{C}-2$ and $\mathrm{C}-3$ carbons of chalcones by addition of a new $\mathrm{CH}_{2}$ group (Scheme 1).

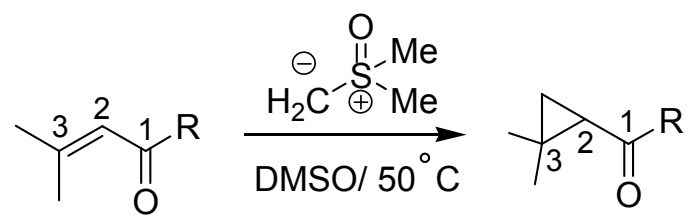

Scheme 1. Corey-Chaykovsky reaction.

In the present work, we wish to report a novel one-pot procedure to synthesis the cyclopropane derivatives with connecting the C-1 and C-3 carbons of chalcones (Scheme 2).

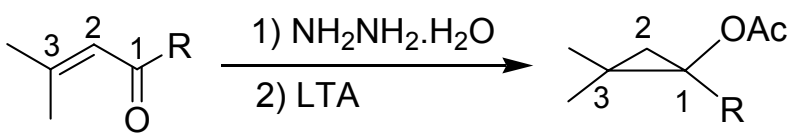

Scheme 2. One-pot procedure for synthesis of cyclopropane from chalcones.

The chalcones 1a-e were prepared by the reaction of indenoquinoxaline with acetophenones in a solvent free condition catalyzed by dimethylamine and then glacial acetic acid and $\mathrm{HCl}{ }^{10,14}$ These chalcones reacted with hydrazine hydrate in toluene and then in situ with lead(IV) acetate (LTA or $\left.\mathrm{Pb}(\mathrm{OAc})_{4}\right)$ to afford new spiro indenoquinoxaline-cyclopropane derivatives 2 a-e and 3a-e (Scheme 3). 

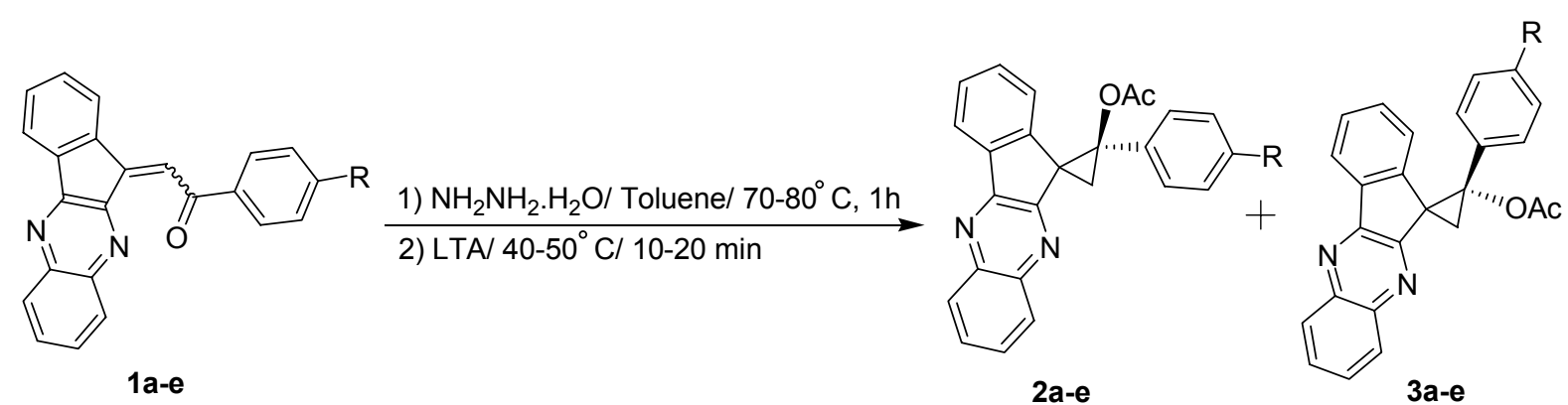

a: $\mathrm{R}=\mathrm{H}, \mathbf{b}: \mathrm{R}=\mathrm{Cl}, \mathbf{c}: \mathrm{R}=\mathrm{Br}$

d: $\mathrm{R}=\mathrm{OMe}$, e: $\mathrm{R}=\mathrm{NO}_{2}$

Scheme 3. One-pot synthesis of spiro indenoquinoxaline-cyclopropane derivatives.

The intermediate of this reaction is a spiro indenoquinoxaline-pyrazoline 4 which was not separated and reacted in situ with LTA to form new diastereomers 2a-e and 3a-e and then the overall yield of the reaction increased. A probable mechanism of the one-pot reaction is presented in scheme 4.

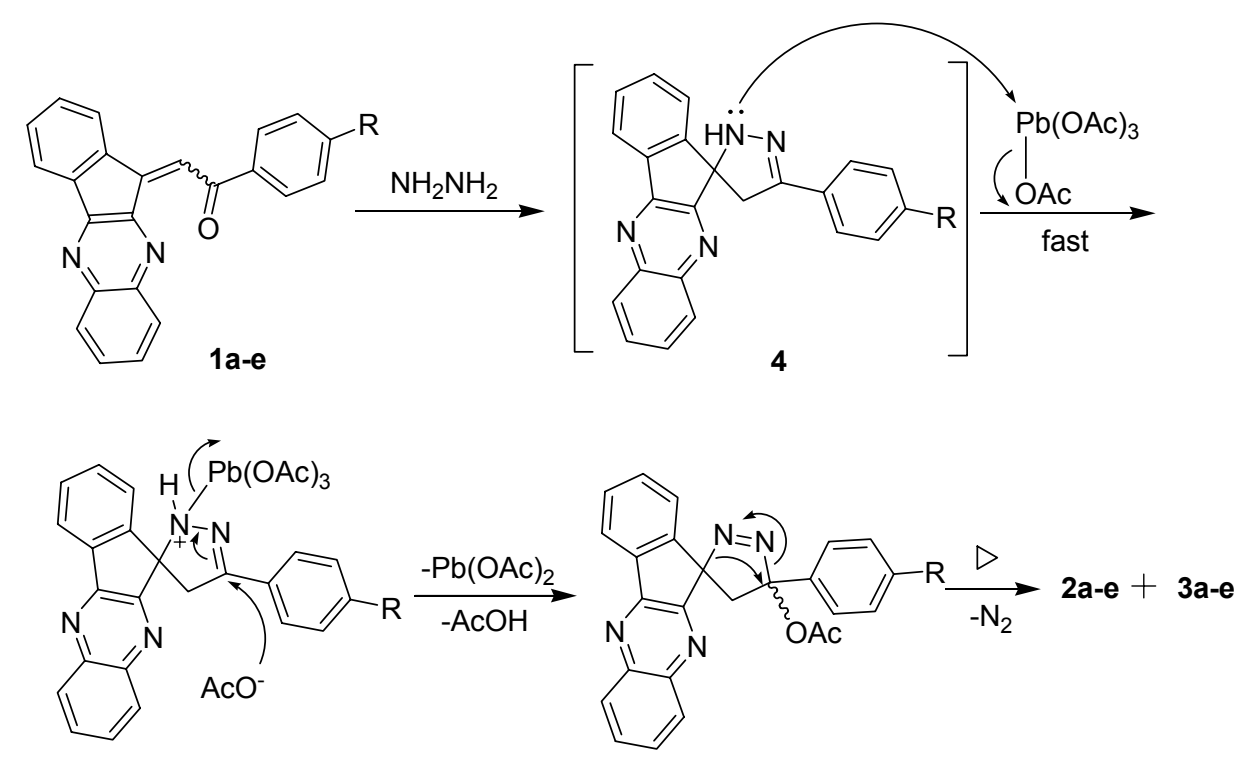

Scheme 4. Reaction mechanism for preparation of diastereomers 2a-e and 3a-e.

This one-pot reaction was diastereoselective and diastereomers 2a-e were prepared in higher yields than their 3a-e isomers. For example, the diastereomeric ratios were determined by integration of separated signals in the ${ }^{1} \mathrm{H}$ NMR spectra of the mixture of compounds $\mathbf{2 a}$ and $\mathbf{3 a}$ in the reaction product (Figure 1). This ratio was $\mathbf{2 a}: \mathbf{3 a}=2.85: 1$. The ratios of other derivatives were $\mathbf{2 b}: \mathbf{3 b}=3: 1, \mathbf{2 c}: \mathbf{3 c}=3.35: 1, \mathbf{2 d}: \mathbf{3 d}=1.04: 1, \mathbf{2 e}: \mathbf{3 e}=3: 1$. 

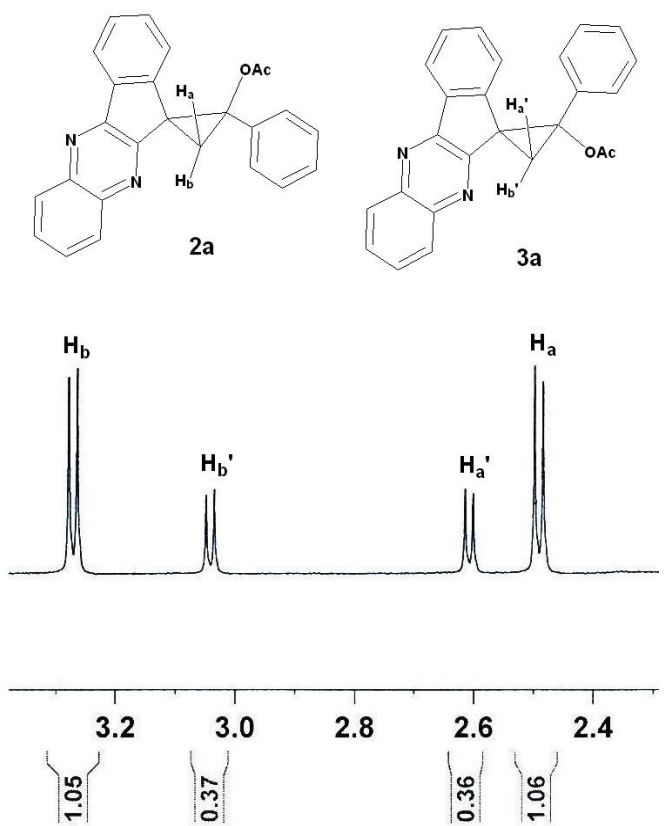

Figure 1. Diastereomeric ratio of $\mathbf{2 a}$ and $\mathbf{3 a}$ determined by ${ }^{1} \mathrm{H}$ NMR signal integrations.

All compounds 2a-e and 3a-e are new derivatives of $11 H$-indeno[1,2- $b$ ]quinoxalin-11-one containing a spiro cyclopropane ring at $\mathrm{C}-11$ carbon atom of indenoquinoxaline. The prepared compounds have shown an excellent agreement between calculated and experimentally obtained data for $\mathrm{CHN}$ elemental analysis. Their structures were deduced from their elemental analyses and their IR, ${ }^{1} \mathrm{H}$ and ${ }^{13} \mathrm{C}$ NMR spectra.

For instant, the ${ }^{1} \mathrm{H}$ NMR spectrum of $2 \mathrm{a}$ indicated two doublets at $\delta 2.48$ and $3.26 \mathrm{ppm}(\mathrm{J}=$ $0.014 \times 500=7 \mathrm{~Hz}$ ) which belong to diastereotopic $\mathrm{CH}_{2}$ protons of $\mathrm{C}-3$ ' position of cyclopropane ring and a singlet at $\delta 2.10 \mathrm{ppm}$ for hydrogens of $\mathrm{CH}_{3}$ in acetate group. The multiplets at $\delta$ 7.19$8.39 \mathrm{ppm}$ showed the aromatic protons. The ${ }^{1} \mathrm{H}$ decoupled ${ }^{13} \mathrm{C}$ NMR spectrum of $\mathbf{2 a}$ exhibited spiro carbon at $\delta 38.69, \mathrm{C}-2$ ' carbon of cyclopropane ring at $\delta 72.17$, carbon of $\mathrm{CH}_{2}$ at $\delta 27.39$, carbon of $\mathrm{CH}_{3}$ at $\delta 21.29$ and carbon of acetate group at $\delta 170.67 \mathrm{ppm}$.

In the ${ }^{1} \mathrm{H}$ NMR spectrum of compound 3a a doublet was appeared at $\delta 6.07(\mathrm{~J}=0.016 \times 500=$ $8 \mathrm{~Hz}$ ) ppm for the $\mathrm{H}-1$ hydrogen atom of indenoquinoxaline ring. This hydrogen was shielded by the magnetic anisotropy effect of the phenyl ring attached to the position 2' of cyclopropane ring (Figure 2). 

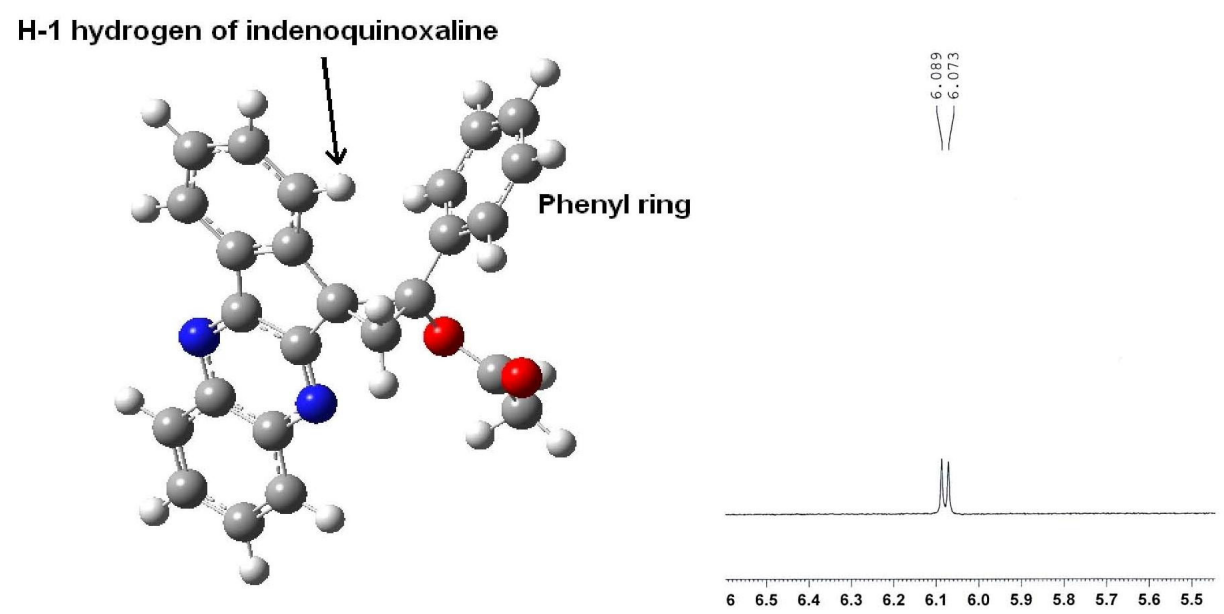

Figure 2. H-1 hydrogen shielded by anisotropic effect of the phenyl ring.

\section{Conclusions}

In summary, some novel spiro indenoquinoxalines containing the cyclopropane ring were synthesized from chalcones in a one-pot simple procedure and the products were obtained in good yields. These compounds can be active biological substances and worthy of attention for the medicinal chemists.

\section{Experimental Section}

General. The chemicals used in this work are the pure products which were purchased from Merck and Fluka companies. Melting points were measured on a Qallenkamp melting point apparatus in open capillary tubes and are uncorrected. IR spectra were taken from a Bruker Vector 22 FT-IR spectrometer. ${ }^{1} \mathrm{H}$ NMR was recorded on a Bruker DRX-400 Avance instrument and ${ }^{13} \mathrm{C}$ NMR $(125 \mathrm{MHz})$ was run on a Bruker DRX-500 Avance instrument using $\mathrm{CDCl}_{3}$ as the solvent and TMS as the internal standard. The purity of prepared spiro compounds was tested by the elemental analysis of $\mathrm{C}, \mathrm{H}$, and $\mathrm{N}$ elements using a Heraus $\mathrm{CHN}$ rapid analyzer. All prepared compounds were filtered and fractionally crystallized from ethanol/water. Indenoquinoxaline were prepared by the procedure described by Ruhemann. ${ }^{13}$ The chalcones of indenoquinoxaline were synthesized with method of Lindwall and Mclennan. ${ }^{14}$

\section{General procedure for preparation of chalcones of indenoquinoxaline.}

To a solid homogenous mixture of $10 \mathrm{mmol}$ indenoquinoxaline and $10 \mathrm{mmol}$ acetophenones, 10 drops of dimethylamine was added and the mixture stirred for 15-30 minutes and a colorless solid formed and then $20 \mathrm{ml}$ glacial acetic acid and five drops of concentrated $\mathrm{HCl}$ was added to 
this precipitate and the mixture warmed in $80{ }^{\circ} \mathrm{C}$ for 30 minutes and after dehydration, chalcones 1a-e were produced and washed with water $(2 \times 20 \mathrm{ml})$ and then recrystallized from absolute ethanol. $^{10}$

\section{General procedure for preparation of spiro indenoquinoxaline-cyclopropane derivatives 2} and 3.

The chalcones 1a-e $(10 \mathrm{mmol})$ were dissolved in $20 \mathrm{ml}$ toluene and then $11 \mathrm{mmol}$ hydrazine hydrate was added to this solution and the mixture was stirred and refluxed at $70-80{ }^{\circ} \mathrm{C}$ for one hour. Then $11 \mathrm{mmol}$ of solid LTA was added to the reaction mixture at $40-50{ }^{\circ} \mathrm{C}$ and nitrogen extrusion began. The reaction was continued for 10-20 minutes and the spiro compounds 2a-e and 3a-e were prepared (Scheme 3). The products were filtered and fractionally crystallized from ethanol/water.

2'-Acetoxy-2'-phenylspiro[indeno[1,2-b]quinoxalin-11,1'-cyclopropane] (2a). Light yellow solid, yield 74\%, decomp.>110 ${ }^{\circ} \mathrm{C}$; IR (KBr): 3052, 2920, 2851, 1752, 1606, $1569 \mathrm{~cm}^{-1}$; ${ }^{1} \mathrm{H}$ NMR (500 MHz) $\delta: 2.10\left(\mathrm{~s}, 3 \mathrm{H}, \mathrm{CH}_{3}\right), 2.48\left(\mathrm{~d}, 1 \mathrm{H}, J=7 \mathrm{~Hz}, \mathrm{CH}_{2 \mathrm{a}}\right), 3.26(\mathrm{~d}, 1 \mathrm{H}, J=7 \mathrm{~Hz}$, $\mathrm{CH}_{2 \mathbf{b}}$ ), 7.19-8.39 (m,13H, ArH); ${ }^{13} \mathrm{C}$ NMR (125 MHz) $\delta: 21.29\left(\mathrm{CH}_{3}\right), 27.39\left(\mathrm{CH}_{2}\right), 38.69$ (spiro carbon), 72.17 (Ph-C-OAc), 122.58, 123.15, 127.98, 128.75, 129.14, 129.25, 129.55, 129.63, $129.76,130.90,131.94,134.89,137.40,141.60,142.45,145.51,154.40,159.63,170.67(\mathbf{C}=\mathrm{O})$; Anal. Calcd. for $\mathrm{C}_{25} \mathrm{H}_{18} \mathrm{~N}_{2} \mathrm{O}_{2}$ (378.4): C, 79.35; H, 4.79; N, 7.40; Found: C, 79.27; H, 4.69; N, $7.38 \%$.

2'-Acetoxy-2'-phenylspiro[indeno[1,2-b]quinoxalin-11,1'-cyclopropane] (3a). Light yellow solid, yield 26\%, decomp. $>110^{\circ} \mathrm{C}$; IR (KBr): 3053, 2920, 2850, 1750, 1608, $1567 \mathrm{~cm}^{-1}$; ${ }^{1} \mathrm{H}$ NMR $(500 \mathrm{MHz}) \delta: 2.01\left(\mathrm{~s}, 3 \mathrm{H}, \mathrm{CH}_{3}\right), 2.60\left(\mathrm{~d}, 1 \mathrm{H}, J=7 \mathrm{~Hz}, \mathrm{CH}_{2 \mathbf{a}}\right), 3.03(\mathrm{~d}, 1 \mathrm{H}, J=7 \mathrm{~Hz}, \mathrm{CH} 2 \mathbf{b})$, 6.07 (d, 1H, $J=8 \mathrm{~Hz}, \mathrm{H}-1$ Indqn.), 7.12-8.34 (m,12H, ArH); ${ }^{13} \mathrm{C}$ NMR (125 MHz) $\delta: 21.44$ $\left(\mathrm{CH}_{3}\right), 26.59\left(\mathrm{CH}_{2}\right), 38.37$ (spiro carbon), 72.13 (Ph-C-OAc), 123.01, 123.39, 128.10, 128.75, 128.86, 129.36, 129.61, 129.76, 130.64, 131.05, 131.94, 134.81, 137.94, 141.24, 142.12, 145.07, 154.09, 158.8, $170.2(\mathbf{C}=\mathrm{O})$; Anal. Calcd. for $\mathrm{C}_{25} \mathrm{H}_{18} \mathrm{~N}_{2} \mathrm{O}_{2}$ (378.4): C, 79.35; H, 4.79; N, 7.40; Found: C, 79.39; H, 4.61; N, 7.30\%.

2'-Acetoxy-2'-(4-chlorophenyl)spiro[indeno[1,2-b]quinoxalin-11,1'-cyclopropane] (2b). Light yellow solid, yield $75 \%$, decomp. $>107{ }^{\circ} \mathrm{C}$; IR (KBr): 3060, 2924, 2851, 1756, 1610, 1588 $\mathrm{cm}^{-1}$; ${ }^{1} \mathrm{H}$ NMR (400 MHz) $\delta: 2.09\left(\mathrm{~s}, 3 \mathrm{H}, \mathrm{CH}_{3}\right), 2.46\left(\mathrm{~d}, 1 \mathrm{H}, J=7 \mathrm{~Hz}, \mathrm{CH}_{2 \mathrm{a}}\right), 3.19(\mathrm{~d}, 1 \mathrm{H}, J=7$ $\left.\mathrm{Hz}, \mathrm{CH}_{2 \mathbf{b}}\right), 7.16-8.33$ (m, 12H, ArH); ${ }^{13} \mathrm{C} \mathrm{NMR}(125 \mathrm{MHz}) \delta: 21.38\left(\mathrm{CH}_{3}\right), 26.60\left(\mathrm{CH}_{2}\right), 38.34$ (spiro carbon), 71.24 (Ar-C-OAc), 123.07, 123.36, 128.21, 128.26, 129.03, 129.07, 129.32, 129.40, 129.64, 131.11, 132.28, 133.50, 137.97, 141.19, 142.10, 144.73, 154.07, 158.45, 170.02 $(\mathbf{C}=\mathrm{O})$; Anal. Calcd. for $\mathrm{C}_{25} \mathrm{H}_{17} \mathrm{ClN}_{2} \mathrm{O}_{2}$ (412.9): C, 72.73; H, 4.15; N, 6.79; Found: C, 72.65; H, $4.03 ; \mathrm{N}, 6.77 \%$.

2'-Acetoxy-2'-(4-chlorophenyl)spiro[indeno[1,2-b]quinoxalin-11,1'-cyclopropane]

(3b).

Light yellow solid, yield $25 \%$, decomp. $>107{ }^{\circ} \mathrm{C}$; IR (KBr): 3062, 2925, 2854, 1757, 1613, 1584 $\mathrm{cm}^{-1} ;{ }^{1} \mathrm{H}$ NMR $(400 \mathrm{MHz}) \delta: 1.99\left(\mathrm{~s}, 3 \mathrm{H}, \mathrm{CH}_{3}\right), 2.52\left(\mathrm{~d}, 1 \mathrm{H}, J=7 \mathrm{~Hz}, \mathrm{CH}_{2 \mathrm{a}}\right), 3.01(\mathrm{~d}, 1 \mathrm{H}, J=7$ $\left.\mathrm{Hz}, \mathrm{CH}_{2 \mathbf{b}}\right), 6.10$ (d, 1H, $J=8 \mathrm{~Hz}, \mathrm{H}-1$ Indqn.), 7.14-8.27 (m, 11H, ArH); ${ }^{13} \mathrm{C}$ NMR $(125 \mathrm{MHz}) \delta$ : 
$21.21\left(\mathrm{CH}_{3}\right), 27.26\left(\mathrm{CH}_{2}\right), 38.64$ (spiro carbon), 71.24 (Ar-C-OAc), 122.74, 125.72, 127.98, $128.65,129.24,129.36,129.46,129.53,129.78,130.80,132.28,133.38,137.49,141.56,142.48$, 145.11, 154.33, 159.34, $170.69(\mathbf{C}=\mathrm{O})$; Anal. Calcd. for $\mathrm{C}_{25} \mathrm{H}_{17} \mathrm{ClN}_{2} \mathrm{O}_{2}$ (412.9): $\mathrm{C}, 72.73$; $\mathrm{H}$, 4.15; N, 6.79; Found: C, 72.59; H, 4.11; N, 6.64\%.

2'-Acetoxy-2'-(4-bromophenyl)spiro[indeno[1,2-b]quinoxalin-11,1'-cyclopropane]

(2c).

Light yellow solid, yield $77 \%$, decomp. $>150^{\circ} \mathrm{C}$; IR (KBr): 3060, 2928, 2849, 1751, 1612, 1550 $\mathrm{cm}^{-1} ;{ }^{1} \mathrm{H}$ NMR (400 MHz) $\delta: 2.09\left(\mathrm{~s}, 3 \mathrm{H}, \mathrm{CH}_{3}\right), 2.45\left(\mathrm{~d}, 1 \mathrm{H}, J=7 \mathrm{~Hz}, \mathrm{CH}_{2 \mathrm{a}}\right), 3.18(\mathrm{~d}, 1 \mathrm{H}, J=7$ $\left.\mathrm{Hz}, \mathrm{CH}_{2 \mathbf{b}}\right), 7.24-8.33$ (m, 12H, ArH); ${ }^{13} \mathrm{C} \mathrm{NMR}(125 \mathrm{MHz}) \delta: 21.37\left(\mathrm{CH}_{3}\right), 26.55\left(\mathrm{CH}_{2}\right), 38.30$ (spiro carbon), 71.28 (Ar-C-OAc), 123.07, 123.37, 128.27, 129.09, 129.33, 129.40, 129.65, $129.72,131.17,132.00,132.56,133.71,137.98,141.19,142.10,144.70,154.06,158.42,170.01$ $(\mathbf{C}=\mathrm{O})$; Anal. Calcd. for $\mathrm{C}_{25} \mathrm{H}_{17} \mathrm{BrN}_{2} \mathrm{O}_{2}$ (457.3): C, 65.66; H, 3.75; N, 6.13; Found: C, 65.48; H, $3.66 ; \mathrm{N}, 6.07 \%$.

2'-Acetoxy-2'-(4-bromophenyl)spiro[indeno[1,2-b]quinoxalin-11,1'-cyclopropane] (3c). Light yellow solid, yield $23 \%$, decomp. $>150{ }^{\circ} \mathrm{C}$; IR (KBr): 3059, 2925, 2851, 1751, 1611, 1553 $\mathrm{cm}^{-1} ;{ }^{1} \mathrm{H}$ NMR (400 MHz) $\delta: 1.99\left(\mathrm{~s}, 3 \mathrm{H}, \mathrm{CH}_{3}\right), 2.51\left(\mathrm{~d}, 1 \mathrm{H}, J=7 \mathrm{~Hz}, \mathrm{CH}_{2 \mathrm{a}}\right), 3.00(\mathrm{~d}, 1 \mathrm{H}, J=7$ $\left.\mathrm{Hz}, \mathrm{CH}_{2 \mathbf{b}}\right), 6.12$ (d, 1H, $J=8 \mathrm{~Hz}, \mathrm{H}-1$ Indqn.), 7.19-8.27 (m, 11H, ArH); ${ }^{13} \mathrm{C}$ NMR (125 MHz) $\delta$ : $21.20\left(\mathrm{CH}_{3}\right), 27.20\left(\mathrm{CH}_{2}\right), 38.45$ (spiro carbon), 71.29 (Ar-C-OAc), 122.74, 122.89, 127.98, $129.23,129.35,129.47,129.52$, 129.78, 130.82, 132.29, 132.56, 133.90, 137.50, 141.70, 142.60, 145.08, 154.45, 159.32, $170.55(\mathbf{C}=\mathrm{O})$; Anal. Calcd. for $\mathrm{C}_{25} \mathrm{H}_{17} \mathrm{BrN}_{2} \mathrm{O}_{2}$ (457.3): C, 65.66; H, 3.75 ; N, 6.13; Found: C, 65.59; H, 3.71; N, 6.02\%.

2'-Acetoxy-2'-(4-methoxyphenyl)spiro[indeno[1,2-b]quinoxalin-11,1'-cyclopropane] (2d). Light yellow solid, yield 51\%, decomp. $>132{ }^{\circ} \mathrm{C}$; IR (KBr): 3060, 2957, 2930, 2836, 1753, 1612 , $1575 \mathrm{~cm}^{-1} ;{ }^{1} \mathrm{H}$ NMR (400 MHz) $\delta: 2.08\left(\mathrm{~s}, 3 \mathrm{H}, \mathrm{CH}_{3}\right), 2.46\left(\mathrm{~d}, 1 \mathrm{H}, J=7 \mathrm{~Hz}, \mathrm{CH}_{2 \mathrm{a}}\right), 3.18(\mathrm{~d}, 1 \mathrm{H}$, $\left.J=7 \mathrm{~Hz}, \mathrm{CH}_{2 \mathbf{b}}\right), 3.81\left(\mathrm{~s}, 3 \mathrm{H}, \mathrm{OCH}_{3}\right), 6.80(\mathrm{~d}, 2 \mathrm{H}, J=8 \mathrm{~Hz}, \mathrm{ArH}), 7.22-8.27(\mathrm{~m}, 10 \mathrm{H}, \mathrm{ArH}) ;{ }^{13} \mathrm{C}$ NMR (125 MHz) $\delta$ : $21.31\left(\mathrm{CH}_{3}\right), 26.92\left(\mathrm{CH}_{2}\right), 38.53$ (spiro carbon), $55.63\left(\mathrm{OCH}_{3}\right), 71.98(\mathrm{Ar}-$ C-OAc), 113.35, 123.00, 123.27, 128.02, 128.84, 129.10, 129.36, 129.74, 130.68, 132.38, 133.42, 137.88, 141.28, 142.04, 145.25, 154.13, 158.94, 159.89, $170.73(\mathbf{C}=\mathrm{O})$; Anal. Calcd. for $\mathrm{C}_{26} \mathrm{H}_{20} \mathrm{~N}_{2} \mathrm{O}_{3}$ (408.4): C, 76.45; H, 4.94; N, 6.86; Found: C, 76.34; H, 4.93; N, 6.83\%.

2'-Acetoxy-2'-(4-methoxyphenyl)spiro[indeno[1,2-b]quinoxalin-11,1'-cyclopropane] (3d). Light yellow solid, yield 49\%, decomp. $>132{ }^{\circ} \mathrm{C}$; IR (KBr): 3061, 2958, 2930, 2834, 1752, 1610, $1572 \mathrm{~cm}^{-1} ;{ }^{1} \mathrm{H}$ NMR (400 MHz) $\delta: 1.98\left(\mathrm{~s}, 3 \mathrm{H}, \mathrm{CH}_{3}\right), 2.52\left(\mathrm{~d}, 1 \mathrm{H}, J=7 \mathrm{~Hz}, \mathrm{CH}_{2 \mathrm{a}}\right), 2.99(\mathrm{~d}, 1 \mathrm{H}, J$ $\left.=7 \mathrm{~Hz}, \mathrm{CH}_{2 \mathbf{b}}\right), 3.73$ (s, 3H, $\left.\mathrm{OCH}_{3}\right), 6.11$ (d, 1H, $J=8 \mathrm{~Hz}, \mathrm{H}-1 \mathrm{Indqn}$ ), 6.70 (d, 2H, $J=8 \mathrm{~Hz}$, $\mathrm{ArH}), 7.12-8.33(\mathrm{~m}, 9 \mathrm{H}, \mathrm{ArH}) ;{ }^{13} \mathrm{C}$ NMR (125 MHz) $\delta: 21.47\left(\mathrm{CH}_{3}\right), 27.77\left(\mathrm{CH}_{2}\right), 38.83$ (spiro carbon), $55.69\left(\mathrm{OCH}_{3}\right), 71.87$ (Ar-C-OAc), 113.99, 121.39, 123.30, 127.70, 128.65, 129.19, 129.46, 129.74, 131.03, 131.76, 132.01, 137.38, 141.59, 142.41, 145.69, 154.42, 159.8, 160.44, $170.07(\mathbf{C}=\mathrm{O})$; Anal. Calcd. for $\mathrm{C}_{26} \mathrm{H}_{20} \mathrm{~N}_{2} \mathrm{O}_{3}$ (408.4): C, 76.45; H, 4.94; $\mathrm{N}, 6.86$; Found: C, $76.41 ; \mathrm{H}, 4.87 ; \mathrm{N}, 6.78 \%$.

2'-Acetoxy-2'-(4-nitrophenyl)spiro[indeno[1,2-b]quinoxalin-11,1'-cyclopropane] (2e). Light yellow solid, yield 75\%, decomp. $>119{ }^{\circ} \mathrm{C}$; IR (KBr): 3060, 2923, 2851, 1755, 1604, $1522 \mathrm{~cm}^{-1}$; ${ }^{1} \mathrm{H}$ NMR (400 MHz) $\delta: 2.14\left(\mathrm{~s}, 3 \mathrm{H}, \mathrm{CH}_{3}\right), 2.50$ (d, $\left.1 \mathrm{H}, J=7 \mathrm{~Hz}, \mathrm{CH}_{2 \mathrm{a}}\right), 3.28(\mathrm{~d}, 1 \mathrm{H}, J=7 \mathrm{~Hz}$, 
$\mathrm{CH}_{2 \mathbf{b}}$ ), 7.14-8.32 (m, 12H, ArH); ${ }^{13} \mathrm{C} \mathrm{NMR}$ (125 MHz) $\delta: 21.27\left(\mathrm{CH}_{3}\right), 26.45\left(\mathrm{CH}_{2}\right), 38.48$ (spiro carbon), 70.43 (Ar-C-OAc), 123.22, 123.54, 124.00, 128.59, 129.33, 129.46, 129.48, 129.66, 131.23, 131.57, 133.02, 138.13, 141.01, 142.17, 144.04, 147.92, 153.99, 157.79, $170.03(\mathbf{C}=\mathrm{O})$;

Anal. Calcd. for $\mathrm{C}_{25} \mathrm{H}_{17} \mathrm{~N}_{3} \mathrm{O}_{4}$ (423.4): C, 70.91; H, 4.05; N, 9.92; Found: C, 70.86; H, 3.97; N, $9.96 \%$.

2'-Acetoxy-2'-(4-nitrophenyl)spiro[indeno[1,2-b]quinoxalin-11,1'-cyclopropane] (3e). Light yellow solid, yield $25 \%$, decomp. $>119{ }^{\circ} \mathrm{C}$; IR (KBr): 3060, 2922, 2851, 1757, 1605, $1524 \mathrm{~cm}^{-1}$; ${ }^{1} \mathrm{H}$ NMR (400 MHz) $\delta: 2.01\left(\mathrm{~s}, 3 \mathrm{H}, \mathrm{CH}_{3}\right), 2.59$ (d, $\left.1 \mathrm{H}, J=7 \mathrm{~Hz}, \mathrm{CH}_{2 \mathrm{a}}\right), 3.07$ (d, $1 \mathrm{H}, J=7 \mathrm{~Hz}$, $\left.\mathrm{CH}_{2 \mathbf{b}}\right), 6.05$ (d, 1H, $J=8 \mathrm{~Hz}, \mathrm{H}-1$ Indqn.), 7.15-8.40 (m, 11H, ArH); ${ }^{13} \mathrm{C} \mathrm{NMR}(125 \mathrm{MHz}) \delta$ : $21.10\left(\mathrm{CH}_{3}\right), 26.77\left(\mathrm{CH}_{2}\right), 38.66$ (spiro carbon), 70.60 (Ar-C-OAc), 123.18, 123.82, 125.81, $128.56,129.42,129.58,129.84,129.93,130.89,131.44,132.90,138.25,141.54,141.66,144.39$, 148, 155.12, 158.20, $170.00(\mathbf{C}=\mathrm{O})$; Anal. Calcd. for $\mathrm{C}_{25} \mathrm{H}_{17} \mathrm{~N}_{3} \mathrm{O}_{4}$ (423.4): C, 70.91; $\mathrm{H}, 4.05 ; \mathrm{N}$, 9.92; Found: C, 70.93; H, 4.05; N, 9.90\%.

\section{Acknowledgements}

We gratefully acknowledge the financial support from the Research Council of Islamic Azad University, Saveh Branch.

\section{References}

1. (a) Baba, Y.; Saha, G.; Nakao, S.; Iwata, C.; Tanaka, T.; Ibuka, T.; Ohishi, H.; Takemoto, Y. J. Org. Chem. 2001, 66, 81. (b) Boger, D. L.; Hughes, T. V.; Hedrick, M. P. J. Org. Chem. 2001, 66, 2207. (c) Graham, D. W.; Ashton, W. T.; Barash, L.; Brown, J. E.; Brown, R. D.; Canning, L. F.; Chen, A.; Springer, J. P.; Rogers, E. F. J. Med. Chem. 1987, 30, 1074. (d) Salaun, J.; Baird, M. S. Curr. Med. Chem. 1995, 2, 511. (e) Yoshida, S.; Rosen, T. C.; Meyer, O. G. J.; Sloan, M. J.; Ye, S.; Haufe, G.; Kirk, K. L. Bioorg. Med. Chem. 2004, 12, 2645. (f) Faust, R. Angew. Chem., Int. Ed. 2001, 40, 2251. (g) Donaldson, W. A. Tetrahedron 2001, 57, 8589. (h) Brandt, W.; Thiemann, T. Chem. Rev. 2003, 103, 1625.

2. (a) Yanovskaya, L. A.; Dombrovsky, V. A.; Khusid, A. Kh. Tsiklopropanis funktsionalnimi gruppami. Sintez i primenenie. (Cyclopropanes with Functional Groups. Synthesis and Application); Nauka: Moscow, 1980. (b) Tsuji, T.; Nishida, S. The Chemistry of the Cyclopropyl Group; Wiley and Sons: New York, NY, 1987. (c) Boche, G.; Walbirsky, H. M. Cyclopropane Derived Intermediates; John Wiley and Sons: New York, NY, 1990. (d) Rappoport, Z. The Chemistry of the Cyclopropyl Group; Wiley and Sons: New York, NY, 1996. (e) Salaün, J. Topics in Current Chemistry; Small Ring Compounds in Organic Synthesis VI: Cyclopropane Derivatives and their Diverse Biological Activities; Springer Berlin: Heidelberg, 2000; Vol. 207, pp 1-67. 
3. Ellis, D.; Kuhen, K. L.; Anaclerio, B.; Wu, B.; Wolff, K.; Yin, H.; Bursulaya, B.; Caldwell, J.; Karanewsky, D.; He, Y. Bioorg. Med. Chem. Lett. 2006, 16, 4246.

4. Yong, S. R.; Ung, A. T.; Pyne, S. G.; Skelton B. W.; White, A. H. Tetrahedron 2007, 63, 1191.

5. (a) Krapcho, A. P. Arkivoc 2007, (ii), 1-53. (b) Ziyat, H.; Ait Itto, M. Y.; Ait Ali, M.; Riahi, A.; Karim, A.; Daran, J.-C. Arkivoc 2006, (xii), 152. (c) Doyle, M. P.; Yan, M. Arkivoc 2002, (viii), 180. (d) Doyle, M. P.; Hu, W. Arkivoc 2003, (vii), 15. (e) Barluenga, J.; Muñiz, K.; Ballesteros, A.; Martínez, S.; Tomás, M. Arkivoc 2002, (v), 110. (f) Cruz, D. C.; Yuste, f.; Díaz, E.; Ortiz, B.; Sánchez-Obregón, S.; Walls, F.; García Ruano, J. L. Arkivoc 2005, (vi), 211.

6. (a) Brock, E. D.; Lewis, D. M.; Yousaf, T. I.; Harper, H. H. (The Procter \& Gamble Company,USA) 1999, WO 991688. (b) Gazit, A.; App, H.; McMahon, G.; Chen, J.; Levitzki, A.; Bohmer, F. D. Med. Chem. 1996, 39, 2170. (c) Sehlstedt, U.; Aich, P.; Bergman, J.; Vallberg, EI.; Norden, B.; Graslund, A. J. Mol. Biol. 1998, 278, 3156.

7. (a) Trillo, R. P. Univ. Microfilms (Ann. Arbor. Mich.), L. C. card no. Mic. 59-4672, 106, Dissertation Abstr. 1959, 20, 1597. (b) Jeney, A.; Zsolnai, T. Zentr. Bakteriol. Parasitenk. Abt. I orig. 1960, 177, 220.

8. (a) Elinson, M. N.; Feducovich, S. K.; Vereshchagin, A. N.; Gorbunov, S. V.; Belyakov, P. A.; Nikishin, G. I. Tetrahedron Lett. 2006, 47, 9129. (b) Elinson, M. N.; Feducovich, S. K.; Stepanov, N. O.; Vereshchagin, A. N.; Nikishin, G. I. Tetrahedron 2008, 64, 708.

9. Azarifar, D.; Shaabanzadeh, M. Molecules 2002, 7, 885.

10. Azizian, J.; Shaabanzadeh M.; Hatamjafari, F.; Mohammadizadeh, M. R. Arkivoc 2006, (xi), 47.

11. Corey, E. J.; Chaykovsky, M. J. Am. Chem. Soc. 1965, 87, 1353.

12. Paxton, R. J.; Taylor, R. J. K. Synlett 2007, 633.

13. Ruhemann S. J. Chem. Soc. 1910, 97, 1438.

14. Lindwall, H. G.; Maclennan, J. S. J. Am. Chem. Soc. 1932, 54, 4739. 